\title{
Celebrating 50 Years of the Turing Award
}

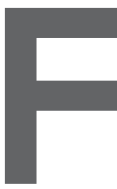

IFTY YEARS AGO, ACM awarded the first A.M. Turing Award to Alan Perlis for his work on advanced programming techniques and compiler construction. Since then, the award has been given annually, with the $50^{\text {th }}$ Turing Award presented last June to Whitfield Diffie and Martin Hellman for their work on public key encryption. In total, 64 men and women from around the world have received the Turing Award, recognizing work laying down the foundations of modern computing.

The prominence of the ACM Turing Award matches the impact of the contributions it honors. In the 50 years since its inception, the Award has become known as the "Nobel Prize of Computing:" Thanks to the generous support of Google, the award currently carries a $\$ 1$ million prize.

To celebrate the first 50 years of the Turing Award, ACM is sponsoring a yearlong series of programs, as highlighted on the Turing $50^{\text {th }}$ website http://www. acm.org/turing-award-50. This site consolidates information about the Turing Laureates and Alan Turing himself. It also presents the Panels in Print and provides information about the upcoming Turing 50th conference.

Panels in Print is a series of writings on key computing topics of the day. The first of these panels features Raj Reddy, Jeff Dean, David Blei, and Pedro Felzenszwalb discussing the state of artificial intelligence and can be found on p. 10 of this issue.

The culminating event of this anniversary year will be ACM's Celebration of 50 Years of the Turing Award conference that will take place June 23-24, 2017, in San Francisco. This event will recognize achievements in computing and will hon- or the Turing Laureates, providing opportunities to hear from many of them. For students and early career members of the computing community there also will be opportunities to meet and converse with the Laureates.

The conference program has been organized around seven moderated panel discussions designed to span a range of computing areas. The goal is to review topics of current interest to both those in the profession as well as to society at large. Those participating in these discussions will include Turing Laureates, ACM award winners, and others involved in shaping the direction of computing. We thank our distinguished Program Committee (Craig Partridge, Fahad Dogar, Karen Breitman, Vint Cerf, Jeff Dean, Joan Feigenbaum, Wendy Hall, Joseph Konstan, and David Patterson) who guided the choice of topics, moderators, and panelists. Panel topics will include:

- Advances in Deep Neural Networks: How are deep neural networks changing our world and our jobs and what breakthroughs maywe imagine going forward?

- Restoring Personal Privacy without Compromising National Security: Can computing technology promote both personal privacy and national security?

- Moore's Law Is Really Dead: What's Next? What old doors will this seismic change close and what new doors will it open?

- Quantum Computing: Far Away? Around the Corner? Or Maybe Both at the Same Time? For both theory and practice, where we are headed, and what quantum skills might be needed by future computing professionals?

- Challenges in Ethics and Computing: How do we recognize and address ethical issues that arise with advances in technology?
- Preserving our Pastfor the Future: How do we archive our electronic artifacts to ensure we can read data and documents in both the near and distant future?

- Augmented Reality-From Gaming to Cognitive Aids and Beyond: How can the sensing and sensory display technologies of augmented reality empower individuals and communities?

There will be multiple opportunities to experience this Turing $50^{\text {th }}$ Celebration event. If you can attend in person you may register at http://www.acm.org/awards/ turing-award-50-conference. There is no registration fee for the meeting, but space will be limited so early registration is essential. Also, I would like to extend a special thank you to several of the SIGs who have sponsored (SIGARCH, SIGCHI, SIGCOMM, SIGGRAPH, SIGHPC, SIGIR, SIGKDD, SIGMM, SIGMOD, SIGPLAN, and SIGSOFT) and supported (SIGACCESS, SIGAI, SIGITE) the Turing event, including funding for students from their SIGs to attend.

If you are not able to attend the event in person, please note that the panel discussions will be streamed live. They also will be video recorded and made available (with subtitles/closed captioning) through the ACM website.

We hope you are able to experience this special ACM activity highlighting the range and impact of Turing Awardwinning work. Whether it be reading the Panels in Print, watching the conference on video, or participating in person in San Francisco, ACM is working to make this a valuable experience.

Vicki L. Hanson (vlh@acm.org) is ACM President, Distinguished Professor at Rochester Institute of Technology, and a professor at the University of Dundee. Twitter: @ACM_President

Copyright held by author. 